\title{
Selecting Adequate Samples for Approximate Decision Support Queries
}

\author{
Amit Rudra ${ }^{1}$, Raj P. Gopalan ${ }^{2}$ and N. R. Achuthan ${ }^{3}$ \\ ${ }^{1}$ School of Information Systems, Curtin University, Ken Street, Bentley WA 6102, Australia \\ ${ }^{2}$ Department of Computing, Curtin University, Ken Street, Bentley WA 6102, Australia \\ ${ }^{3}$ Department of Mathematics and Statistics, Curtin University, Ken Street, Bentley WA 6102, Australia \\ \{A.Rudra, R.Gopalan, N.R.Achuthan\}@curtin.edu.au
}

Keywords: Sampling, Approximate Query Processing, Data Warehousing, Inverse Simple Random Sample without Replacement (SRSWOR).

\begin{abstract}
For highly selective queries, a simple random sample of records drawn from a large data warehouse may not contain sufficient number of records that satisfy the query conditions. Efficient sampling schemes for such queries require innovative techniques that can access records that are relevant to each specific query. In drawing the sample, it is advantageous to know what would be an adequate sample size for a given query. This paper proposes methods for picking adequate samples that ensure approximate query results with a desired level of accuracy. A special index based on a structure known as the k-MDI Tree is used to draw samples. An unbiased estimator named inverse simple random sampling without replacement is adapted to estimate adequate sample sizes for queries. The methods are evaluated experimentally on a large real life data set. The results of evaluation show that adequate sample sizes can be determined such that errors in outputs of most queries are within the acceptable limit of $5 \%$.
\end{abstract}

\section{INTRODUCTION}

Decision support queries often involve applying aggregate functions like count, average, and sum on relatively small subset of records in a data warehouse. Approximate results are usually sufficient for such queries to provide a good idea of how a business is doing. While relatively small random samples can be used effectively for some data warehouse queries, they are not suitable for highly selective queries as the records that satisfy the query conditions may not be adequately represented in such samples. Ideally, an adequate sample for such a query should be drawn from a subset of the data warehouse that satisfies the query's selection conditions. A sample is adequate for a query if it can be used to estimate the query result with its accuracy within a specified confidence interval. In this paper, we focus on estimating the sizes of adequate samples for specific queries.

As Olken and Rotem (1990) pointed out, picking records at random from a database without prior rearrangement is very inefficient since a large number of disk accesses may be needed to retrieve a sample. To alleviate this problem, several recent schemes for approximate query processing have been proposed (Aouich and Lemire 2007); (Heule et al., 2013); (Jermaine 2007); (Jermaine 2003); (Jermaine et al., 2004); (Jin et al., 2006); (Joshi and Jermaine, 2008); (Li et al., 2008); (Spiegel and Polyzotis, 2009). Joshi and Jermaine (2008) introduced the ACE Tree, which is a binary tree index structure for efficiently drawing samples for processing database queries. They demonstrated the effectiveness of this structure for single and two attribute database queries, but did not deal with multi-attribute aggregate queries. For extending the ACE Tree to k key attributes, Joshi and Jermaine (2008) proposed binary splitting of one attribute range after another at consecutive levels of the binary tree starting from the root; from level $\mathrm{k}+1$, the process is repeated with each attribute in the same sequence as before. This process could lead to an index tree of very large height for a data warehouse even if only a relatively small number of attributes are considered. Rudra et al., (2012) proposed the $k$-MDI Tree, that extends the ACE Tree structure to deal with multi-dimensional data warehouses with $k$-ary splits of data ranges. It was shown that random samples of relevant rows (rows 
that satisfy the conditions of a given query) could be drawn more efficiently using the $k$-MDI tree.

The sampling scheme using the $k$-MDI tree index (Rudra et al., 2012) facilitates picking rich samples for queries with highly specific selection conditions. If the sample contains an adequate number of records that satisfy the query conditions, the average values can be estimated from these records. However, to estimate sum (= avg $x$ count) we need to estimate both the average as well as count of the records that satisfy the query in the whole database. Therefore, from the sample we need to project the number of records in the entire database that satisfy the given query conditions. Chaudhuri and Mukerjee (1985) proposed an unbiased estimator based on inverse simple random sampling without replacement (SRSWOR) where random sampling is carried out on a finite population until a predefined number of domain members are observed. In this paper, we propose the adaptation of inverse SRSWOR to estimate adequate sample sizes for queries using the $k$-MDI tree index. The method is empirically evaluated on a large real world data warehouse.

The rest of the paper is organized as follows: In Section 2 , we briefly describe the $k$-way multidimensional $(k$-MDI) indexing structure and the storage structure of data records. Section 3 discusses how to pick adequate samples using inverse SRSWOR. In Section 4 we discuss the results of our experiments. Finally, Section 5 concludes the paper.

\section{TERMS, DEFINITIONS AND $k$-MDI TREE INDEX}

In this section, we define some terms pertaining to data warehousing, define confidence interval and then review the $k$-MDI tree index for retrieving relevant samples from a data warehouse.

\subsection{Dimension and Measures}

To execute decision support queries, data is usually structured in large databases called data warehouses. Typically, data warehouses are relational databases with a large table in the middle called the fact table connected to other tables called dimensions. For example, consider the fact table Sales shown as Table 1. A dimension table Store linked to StoreNo in this fact table will contain more information on each of the stores such as store name, location, state, and country (Hobbs et al., 2003). Other dimension tables could exist for items and date. The remaining attributes like quantity and amount are typically, but not necessarily, numerical and are termed measures. A typical decision support query aggregates a measure using functions such as $\operatorname{Sum}(), \operatorname{Avg}()$ or Count(). The fact table Sales along with all its dimension tables forms a star schema.

Table 1: Fact table SALES.

\begin{tabular}{|c|l|l|c|c|}
\hline \multicolumn{5}{|c|}{ SALES } \\
\hline StoreNo & Date & Item & Quantity & Amount \\
\hline 21 & 12-Jan-11 & iPad & 223 & 123,455 \\
\hline 21 & 12-Jan-11 & PC & 20 & 24,800 \\
\hline 24 & 11-Jan-11 & iMac & 11 & 9,990 \\
\hline 77 & 25-Jan-11 & PC & 10 & 12,600 \\
\hline
\end{tabular}

In decision support queries a measure is of interest for calculation of averages, totals and counts. For example, a sales manager may like to know the total sales quantity and amount for certain item(s) in a certain period of time for a particular store or even all (or some) stores in a region. This may then allow her to make decisions to order more or less stocks as appropriate at a point in time.

\subsection{Multidimensional Indexing}

The $k$-ary multi-dimensional index tree ( $k$-MDI tree) proposed in Rudra et al., (2012) extends the ACE Tree index (Joshi and Jermaine, 2008) for multiple dimensions. The height of the $k$-MDI tree is limited to the number of key attributes. As a multi-way tree index, it is relatively shallow even for a large number of key value ranges and so requires only a small number of disk accesses to traverse from the root to the leaf nodes.

The $k$-MDI tree is a $k$-ary balanced tree (Bentley 1975) as described below:

1. The root node of a $k$-MDI tree corresponds to the first attribute (dimension) in the index.

2. The root points to $k_{1}\left(k_{1} \leq k\right)$ index nodes at level 2 , with each node corresponding to one of the $k_{1}$ splits of the ranges for attribute $a_{1}$.

3. Each of the nodes at level 2, in turn, points to up to $k_{2} \quad\left(k_{2} \leq k\right)$ index nodes at level 3 corresponding to $k_{2}$ splits of the ranges of values of attribute $a_{2}$; similarly for nodes at levels 3 to $h$, corresponding to attributes $a_{3}, \ldots, a_{\mathrm{h}}$.

4. At level $h$, each of up to $k^{h-1}$ nodes points to up to $k_{\mathrm{h}}\left(k_{\mathrm{h}} \leq k\right)$ leaf nodes that store data records.

5. Each leaf node has $h+1$ sections; for sections 1 to $h$, each section $i$ contains random subset of records in the key range of the node $i$ in 


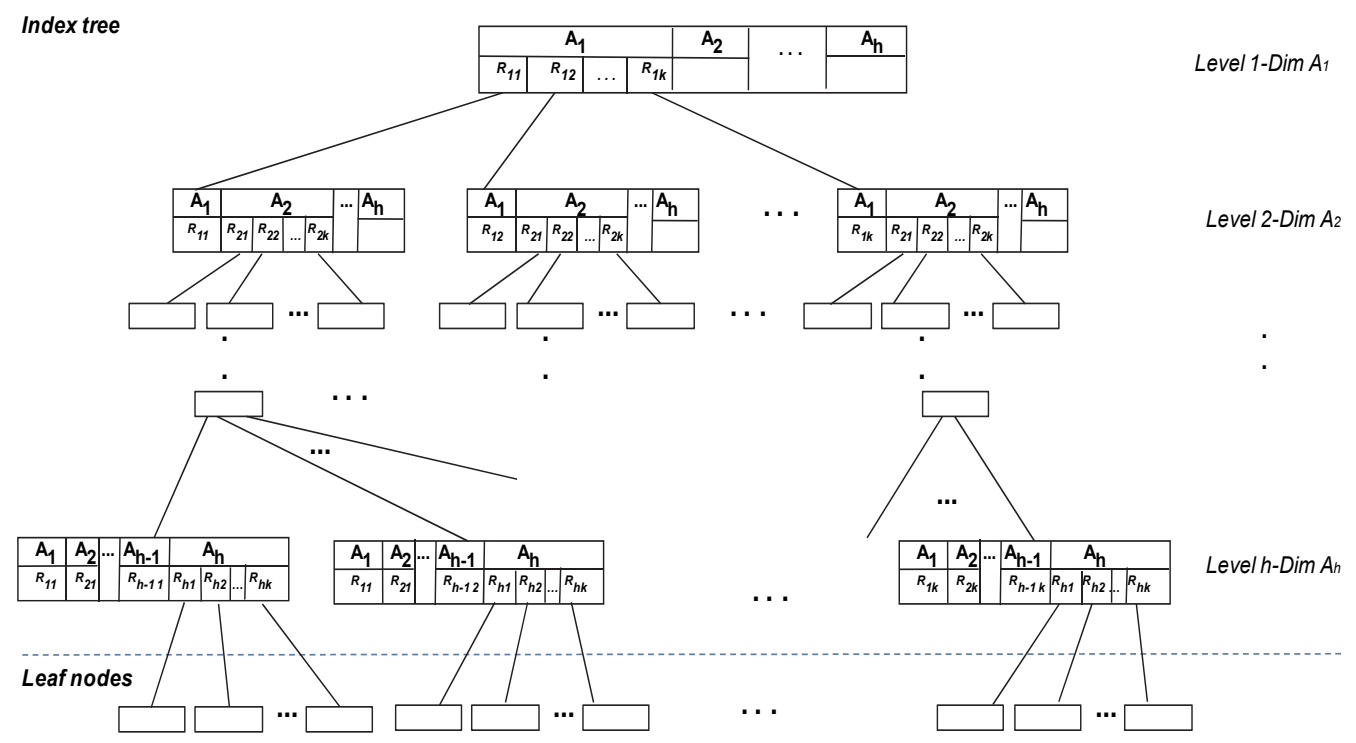

Figure 1: General structure of the k-MDI tree.

the path from the root to the level $h$ above the leaf; section $h+1$ contains a random subset of records with keys in the specific range for the given leaf.

Thus, the dataset is divided into a maximum of $k^{h}$ leaf nodes with each leaf node, in turn, consisting of $h+1$ sections and each section containing a random subset of records. The total number of leaf nodes depends on the total number of records in the dataset and the size of a leaf node (which may be chosen as equal to the disk block size or another suitable size). More details on leaf nodes and sections are given in sub-section II.C. In real data sets, the number of range splits at different nodes of a given level $i$ need not be the same. For convenience, the number of splits at all levels are kept as $k$ in Figure 1 that shows the structure of the general scheme for $k$-MDI multilevel index tree of attributes $\mathrm{A}_{1}, \mathrm{~A}_{2}, \ldots, \mathrm{A}_{\mathrm{h}}$ with $k$ ranges $\left(R_{11}, R_{12}, \ldots, R_{1 \mathrm{k}}\right),\left(R_{21}, R_{22}, \ldots, R_{2 \mathrm{k}}\right), \ldots$ $\left(R_{\mathrm{h} 1}, R_{\mathrm{h} 2}, \ldots, R_{\mathrm{hk}}\right)$ respectively at levels $(1, \ldots, h)$. In other words, $R_{i j}$ is the $i$-th attribute's $j$-th range high water mark (HWM).

An example of the $k$-MDI tree is shown in Figure 1 from a store chain dataset with three dimensions store, date sold and item number. The number of range splits and hence branches from non-leaf nodes vary between 2 and 4 in this example.

\subsection{Leaf Nodes}

The lowest level nodes of a $k$-MDI tree point to leaf nodes containing data records. The data records are stored in $h+1$ sections, where $h$ is the height of the tree. Section $S_{1}$ of every leaf node is drawn from the entire database with no range restriction on the attribute values. Each section $S_{\mathrm{i}}(2 \leq i \leq h+1)$ in a leaf node $L$ is restricted on the range of key values by the same restrictions that apply to the corresponding sub-path along the path from the root to $L$. Thus for section $S_{2}$, the restrictions are the same as on the branch to the node at level 2 along the path from the root to $L$ and so on.

Figure 2 shows an example leaf node projected from the sample $k$-MDI tree. The sections are indicated above the node with attribute ranges for each section below the node. The circled numbers in each section indicate record numbers that are randomly placed in the section. The range restrictions on the records are indicated below each section, where the first section $S_{1}$ has records drawn from the entire range of the database. Thus, it can contain records uniformly sampled from the whole dataset. The next section $S_{2}$ has restriction on the first dimension viz. store (for leaf node $L_{7}$ this range is store numbers 1-16). The third section $S_{3}$ has restrictions on both first and second dimensions viz. store and date. While the last section $S_{4}$ has restrictions on all the three dimensions - store, date and item.

The scheme for selection of records into various leaf nodes and sections is explained in detail in the following section.

\subsection{Using the $k$-MDI Tree for Data Warehouse Queries}

By using a $k$-MDI tree index, we can draw stratified samples for data warehousing queries from restricted 


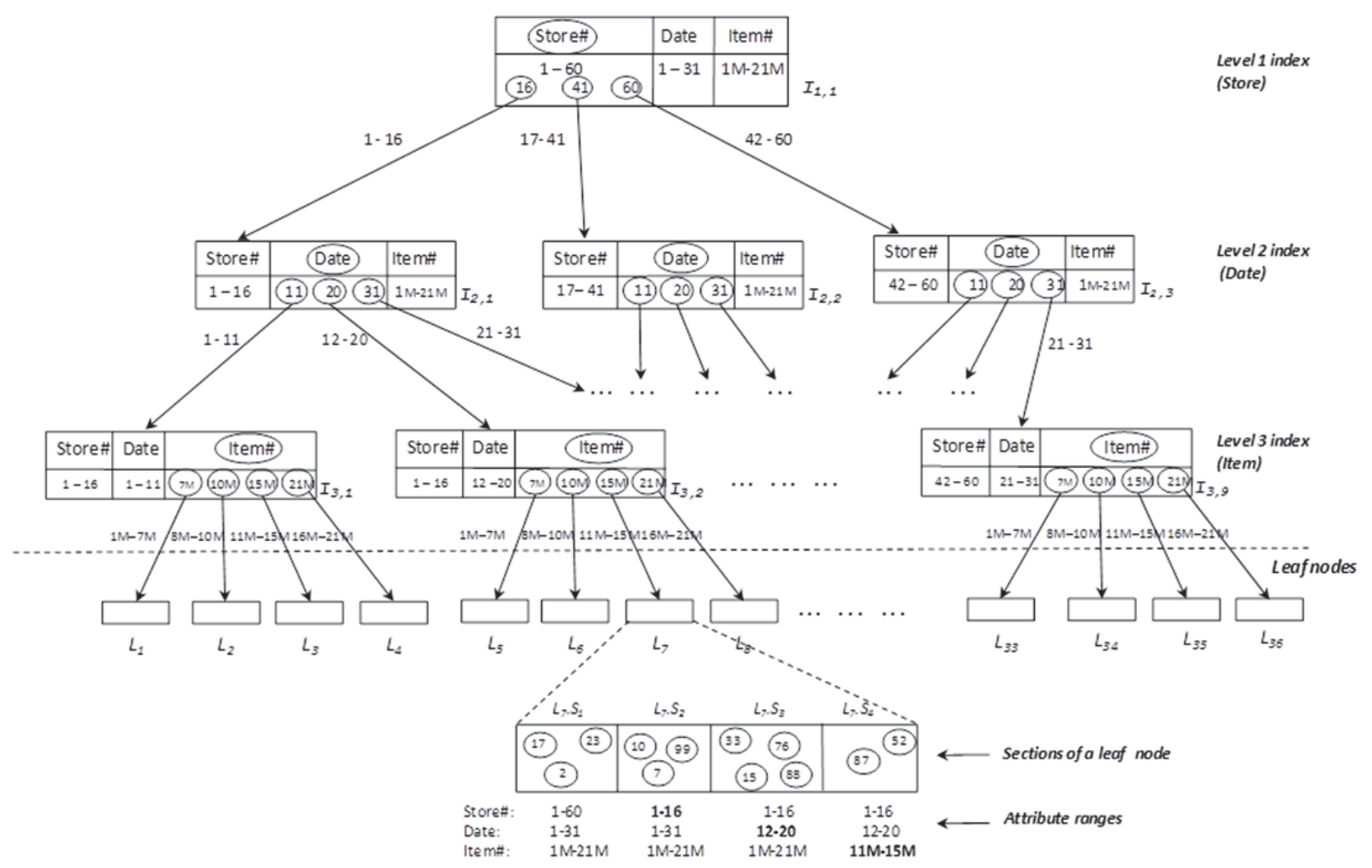

Figure 2: A leaf node (changes in range values for attributes are indicated in bold).

ranges of key values. The database relevancy ratio (DRR) of a query $Q$, denoted by $\rho(Q)$ is the ratio of the number of records in a dataset $\mathrm{D}$ that satisfies the query conditions to the total number of records in $\mathrm{D}$. For a query with no condition, $\rho(Q)$ is 1 . Similarly, the sample relevancy ratio (SRR) of a query $\mathrm{Q}$ for a sample set $S$, denoted by $\rho(Q, S)$ is defined as the ratio of the number of records in $S$ that satisfy a given query $\mathrm{Q}$ to the total number of records in $\mathrm{S}$.

In a true random sample of records, the SRR for a query $\mathrm{Q}$ is expected to be equal to its $\mathrm{DRR}$, i.e., $\mathrm{E}(\rho(\mathrm{Q}, \mathrm{S}))=\rho(\mathrm{Q})$. A sample with $\rho(\mathrm{Q}, \mathrm{S})>\rho(\mathrm{Q})$ is likely to give a better estimate of the mean than a true random sample. However, for the sum of a column, the sample needs to be representative of the population, i.e., $\rho(Q, S)$ should be close to $\rho(Q)$.

Consider the following formula for estimating the sum (Berenson and Levine 1992): $\hat{T}=N \hat{p} \bar{X}$, where $N$ is the cardinality of the population, $\hat{p}$ the estimated proportion of records satisfying the query conditions and $\bar{X}$ the mean of records in the sample satisfying the query condition. In order to estimate the mean we can use all relevant sampled records from all sections of the retrieved leaf nodes, but to estimate the sum we can use sampled records only from section $\mathrm{S}_{1}$, which is the only section with records drawn randomly from the entire dataset. For estimating the sum for a query with conditions on some of the indexed dimensions we use appropriate sections of the retrieved leaf nodes to get a better estimate of the mean; the records from section $\mathrm{S}_{1}$ are used to get a fair estimation of the proportion of records that satisfy the query conditions.

\subsection{Effect of Sectioning on Relevancy Ratio}

As discussed earlier, sections $S_{1}$ to $S_{h+1}$ of each leaf node contain random collections of records with the difference that $S_{1}$ contains records from the entire dataset while other sections contain random records from restricted ranges of the key attributes. Consider a query with the same range restrictions on all three dimensions (store, date and item) as section $L_{7} . S_{4}$ in Figure 2. We are then likely to get more relevant records in the sample from the second section $L_{7} . S_{2}$ than from $S_{1}$ since records of $S_{2}$ have restrictions on the first dimension of store that matches the query condition. Records in $S_{3}$ will have restrictions on both store and date dimensions that match that of the query and so likely to contain more relevant records than in $S_{2}$. All records in section $L_{7} . S_{4}$ will satisfy the query since the range restrictions on $S_{4}$ exactly match the query. Mathematically, for a query $Q$ having restrictions as mentioned above: 


$$
\begin{gathered}
\rho(\mathrm{Q})=\mathrm{E}\left(\rho\left(\mathrm{Q}, L_{7 .} S_{l}\right)\right) \leq \mathrm{E}\left(\rho\left(\mathrm{Q}, L_{7 .} S_{2}\right)\right) \\
\leq \mathrm{E}\left(\rho\left(\mathrm{Q}, L_{7 .} S_{3}\right)\right) \leq \mathrm{E}\left(\rho\left(\mathrm{Q}, L_{7 .} S_{4}\right)\right)
\end{gathered}
$$

Using this property of the $k$-MDI tree, it is possible to quickly increase the size of a sample that is too small, by including more records from other sections of the retrieved leaf nodes.

\subsection{Record Retrieval to Process a Query}

The objective of using the k-MDI tree is to retrieve a significant number of relevant records (i.e. records that satisfy the query conditions) in the sample drawn for processing a given query. The query conditions may span sections of one or more leaf nodes, which can be reached from index nodes that straddle more than one range of attribute values. Traversing the tree from the root using the attribute value ranges in the query conditions can access these leaf nodes. Sections from multiple leaf nodes are then combined to form the sample.

We describe the retrieval process using an example query on the sample database of Figure 2. Consider a query $\mathrm{Q}_{0}$ about sales in store 12 for date range 1-13 and item range $12 \mathrm{M}-20 \mathrm{M}$. The retrieval algorithm finds the sections of leaf nodes for this query as follows:

1. Search index level 1 to locate the relevant store range. Store 12 is in the left most range of 1-16.

2. Traverse down to index level 2 (date), indicated by a dashed arrow in Figure 3, along the first store range. Since there is a condition on date (113), compare the HWMs (high water marks) of the three ranges and find that it fits into two date ranges viz. the first and the second. Make a note of these date ranges.

3. Traverse down using the first date range to the next index level, which has item ranges. Since there is a condition on item numbers (12M20M), compare this range with HWMs and find that it fits into two ranges viz. the third and the fourth. Make a note of these item ranges.

4. Traverse down using the third item range to relevant leaf pages and make a note of them.

5. Iterate step 4 , except this time using the fourth item range.

6. Next, repeat the above three steps i.e. steps 3 through 5; but this time using the second date range instead.

7. Now retrieve records from the relevant sections in the four leaf nodes (viz. $L_{3}, L_{4}, L_{7}$ and $L_{8}$ ) to form a sample for the given query.

\section{SAMPLING TO ADEQUATE LEVELS USING INVERSE SRSWOR}

An important question to be answered is how much sampling is adequate to estimate some property of a population. For business analytics on very large data (Chaudhuri, 2012), it is valuable to provide the analytic query user with incremental feedback of the ongoing progress of an approximate query (Fisher 2011); (Fisher et al., 2012). The method described below for determining if an adequate sample size has been retrieved is based on the works of Chaudhuri and Mukerjee (1985) and Sangngam and Suwatee (2010). Their research established that inverse simple random sampling without replacement (or inverse SRSWOR) method provides an unbiased estimate of the number of data points (M) satisfying property $\mathrm{P}$, if sampling is continued until a pre-assigned number, say $\mathrm{m}$, of data points satisfying property $\mathrm{P}$ are present in the sample. In the present case, property $P$ would be the conditions imposed on a query viz. the dimension ranges. For a given query $\mathrm{Q}$, assume that we have determined the estimate of $\rho(Q)$.

Recall that

$\rho(Q)=\frac{\text { Number of cases satisying conditions of query } \mathrm{Q}}{\text { sample size } \mathrm{n}}$.

We could also choose the above sample size $\mathrm{n}$ as follows:

Fix the error we are ready to tolerate, say e. Then choose $n=\frac{z_{\alpha / 2}^{2} \rho(Q)(1-\rho(Q))}{e^{2}}$, where $e$ is a number such as $0.01,0.02$, or 0.05 etc. and $z_{\alpha / 2}$ is the normal ordinate such that $\operatorname{Prob}\left\{\mathrm{P}(\mathrm{Q})>z_{\alpha / 2}\right\}=\frac{\alpha}{2}$ where $\mathrm{P}(\mathrm{Q})$ is the random variable representing $\rho(Q)$. Usually we take $\alpha=0.05$ for $95 \%$ confidence interval.

Now estimate $\rho(Q)$ again using this new sample size. Henceforth, assume that we have this new estimate with error bounded by $e$. In fact, when the true value of $\rho(Q)$ is not too close to zero $(0)$ or one (1), and sample size $\mathrm{n}$ is large enough we know that the random variable $\mathrm{P}(\mathrm{Q})$ representing $\rho(Q)$, follows approximately the Normal distribution with mean $\rho(Q)$ and standard deviation $\sqrt{\frac{\rho(Q)(1-\rho(Q))}{n}}$. Now assume that the true value of $\rho(Q)$ is not too close to zero $(0)$ or one (1), then we can get $95 \%$ confidence interval for true value of $\rho(Q)$ as 


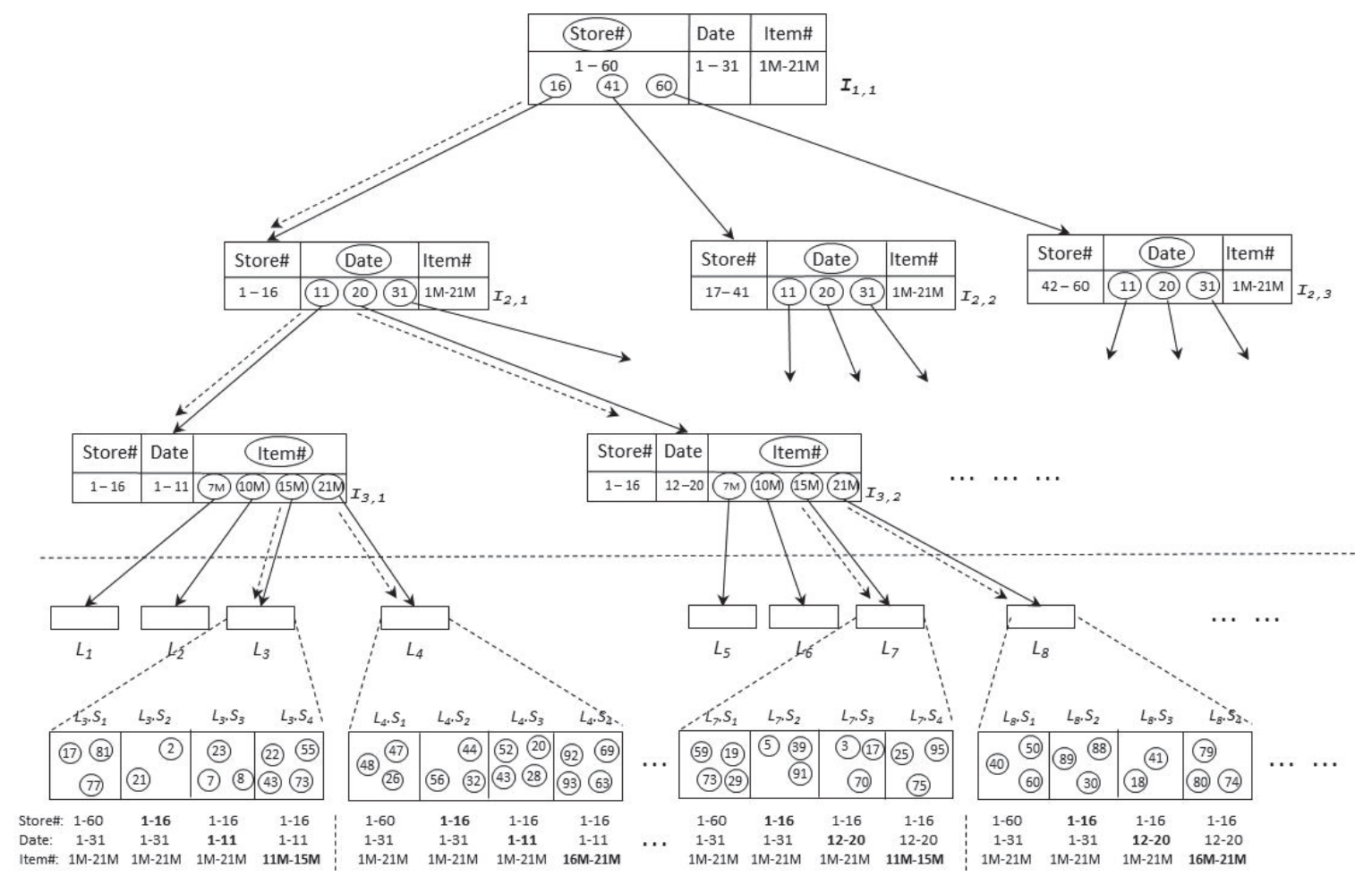

Figure 3: Navigation down index tree nodes for conditions on three dimensions.

$\left(\rho(Q)-1.96 \sqrt{\frac{\rho(Q)(1-\rho(Q))}{n}}, \rho(Q)+1.96 \sqrt{\frac{\rho(Q)(1-\rho(Q))}{n}},\right)$

Using the confidence interval (1) we can choose about 5 to 10 possible values for $\mathrm{P}(\mathrm{Q})$. These values may correspond to $10,25,50,75,90$ percentile points of the random variable $\mathrm{P}(\mathrm{Q})$. Let us denote these values by $\rho_{1}(Q), \rho_{2}(Q), \rho_{3}(Q), \rho_{4}(Q)$ and $\rho_{5}(Q)$.

Using these 5 percentiles, choose $10 \%$ or $20 \%$ of the corresponding $\mathrm{M}$ values as possible choice of values for $\mathrm{m}$. More specifically,

$$
\begin{gathered}
m_{1,1}=0.10 \rho_{1}(Q) N, m_{2,1}=0.20 \rho_{1}(Q) N ; \\
m_{1,2}=0.10 \rho_{2}(Q) N, m_{2,2}=0.20 \rho_{2}(Q) N ; \\
m_{1,3}=0.10 \rho_{3}(Q) N, m_{2,3}=0.20 \rho_{3}(Q) N ; \\
m_{1,4}=0.10 \rho_{4}(Q) N, m_{2,4}=0.20 \rho_{4}(Q) N ; \\
\quad \text { and } \\
m_{1,5}=0.10 \rho_{5}(Q) N, m_{2,5}=0.20 \rho_{5}(Q) N
\end{gathered}
$$

Henceforth, denote these 10 possible values of $\mathrm{m}$ by $m_{t}, 1 \leq t \leq 10$. We now describe the Basic Find_M or $B F \_M$ algorithm and the steps involved. There are five steps in the $B F \_M$ algorithm, which are as follows:

1. Initialization: In this step, various variables, like counts and cumulative totals, are initialized.
2. Sampling: Records are sampled iteratively and those meeting the given condition i.e. satisfying the given property, say $P$, are used for calculating the sum and the count of the value(s) in the query.

3. Check \& Iterate: This step checks if adequate number of samples have been retrieved. In case, it has not reached the targeted number of samples, it iterates the sampling step, else it terminates further sampling.

4. Estimation: In this step, unbiased estimators of $\mathrm{M}$, average and sum, and their variances are computed.

5. Best Estimate: Choose the best estimate as the one that minimizes the desired variance.

The $B F M$ algorithm used for the inverse sampling without replacement is shown in Algorithm 1.

Now for a given query Q, we can use Algorithm 1 to recommend the appropriate value of $m$ that yields the smallest variance for the estimate of $\mathrm{M}$. To determine the number of sections to retrieve, frequency tables for all dimensions are used. In case the query involves more than one dimension, information in frequency tables for all dimensions involved in the query condition is utilized. 


\section{Algorithm 1. Basic Find_M (BF $M)$ Algorithm.}

Input Dataset to be sampled $D$, Cardinality of dataset $\mathrm{N}$, the 10 different values of $\mathrm{m}_{\mathrm{t}}, 1 \leq$ $\mathrm{t} \leq 10$.

Output Estimates of $\mathrm{M}$, average and sum of population.

Choose $\mathrm{m}=\mathrm{m}_{t}, 1 \leq \mathrm{t} \leq 10$ and continue to draw a SRSWOR until the sample has at least $\mathrm{m}_{\mathrm{i}}$ transitions with property Q. More precisely, we use the following steps.

\section{Begin}

1. Initialization step: set $\mathrm{i}=1, \mathrm{n}_{0}=$ current sample size $=0, \mathrm{ml}_{0}=$ the number of transactions satisfying property $Q=0$ and $\operatorname{Sum}_{0}=0$ and $\operatorname{SumX}_{0}^{2}=0$.

Corresponding to query $\mathrm{Q}$, let $\mathrm{L}_{\mathrm{i}}, 1 \leq \mathrm{i} \leq r$ be the list of all leaves of the tree that are candidates for drawing samples.

Set $\mathrm{t}=1$;

While $\mathrm{t} \leq 10$ Loop

Set $m=m_{t}$

2. Sampling step: Choose at random a leaf $\mathrm{Li}$ from the set of $\mathrm{r}$ candidate leaves. From leaf $\mathrm{L}_{\mathrm{i}}$ choose section $\mathrm{S}_{\mathrm{p}}, 1 \leq p \leq 4$ during $\mathrm{p}^{\text {th }}$ visit to this leaf.

Let transactions from $\mathrm{S}_{\mathrm{p}}$ be denoted by $\mathrm{T}_{\mathrm{i} 1}, \mathrm{~T}_{\mathrm{i} 2}$, $\ldots, \mathrm{T}_{\mathrm{iq}}$.

Set $\mathrm{j}=0 ; \mathrm{n}_{\mathrm{i}}=\mathrm{n}_{\mathrm{i}-1} ; \mathrm{ml}_{\mathrm{i}}=\mathrm{m} 1_{\mathrm{i}-1}$ and $\operatorname{Sum}_{\mathrm{i}}=\operatorname{Sum}_{\mathrm{i}-1}$ and $\operatorname{SumX}_{\mathrm{i}}{ }_{\mathrm{i}}=\operatorname{SumX}_{\mathrm{i}-1}^{2}$

Repeat

$$
\mathrm{j}=\mathrm{j}+1 \text {; }
$$

$\mathrm{n}_{\mathrm{i}}=\mathrm{n}_{\mathrm{i}}+1$

If $T_{i(j+1)}$ satisfies the property $P$

then

$\mathrm{m}_{\mathrm{i}}=\mathrm{m}_{\mathrm{i}}+1$

$\operatorname{Sum}_{\mathrm{i}}=\mathrm{Sum}_{\mathrm{i}}+\mathrm{X}_{\mathrm{Tij}}$ and

$\operatorname{SumX}^{2}{ }_{\mathrm{i}}=\operatorname{Sum}_{{ }_{\mathrm{i}}}+\left(\mathrm{X}_{\mathrm{Tij}}\right)^{2}$.

$\operatorname{Until}\left(\mathrm{j}=\mathrm{q}\right.$ or $\left.\mathrm{ml}_{\mathrm{i}}=\mathrm{m}\right)$

3. Check \& Iterate step: If $m 1_{\mathrm{i}}<\mathrm{m}$ then set $\mathrm{i}=$ $\mathrm{i}+1$ and go to step 2. Otherwise, go to step 4 since the sampling procedure has terminated (with reference to current value of $\mathrm{m}$ ) and ready to determine the unbiased estimates of M, Sum and Average (using current value of $\mathrm{m})$.

4. Estimation step: Set sample size $n=n_{i}$ and $m$ $=$ the number of transactions satisfying the property $\mathrm{Q}$ and set sum' $=\mathrm{Sum}_{\mathrm{i}}$ and $\mathrm{Z}=$ $\left(\operatorname{SumX}_{i}{ }_{i}\right) / \mathrm{m}$
Unbiased estimate of $\mathrm{M}=\hat{\mathrm{M}}_{t}=\mathrm{N} \frac{\mathrm{m}-1}{n-1}$

Unbiased estimate of variance of $\widehat{M}_{t}$ is

Unbiased estimate of Average is

$$
\overline{\mathrm{x}}_{t}=\frac{\text { sum' }^{\prime}}{\mathrm{m}}
$$

Unbiased estimate of Sum is

$$
\begin{gathered}
\widehat{\mathrm{T}}_{t}=\hat{\mathrm{M}}_{t} \overline{\mathrm{x}}_{t} \\
\text { Define } \mathrm{s}_{t}^{2}=\frac{\mathrm{m}}{\mathrm{m}-1}\left(\mathrm{Z}-\left(\overline{\mathrm{x}}_{t}\right)^{2}\right)
\end{gathered}
$$

Unbiased estimate of variance of Average is

$$
=\mathrm{v}\left(\overline{\mathrm{x}}_{t}\right)=\frac{\hat{\mathrm{M}}_{t}-\mathrm{m}}{\hat{\mathrm{M}}_{t}(\mathrm{~m}-1)}\left(\mathrm{Z}-\left(\overline{\mathrm{x}}_{t}\right)^{2}\right) .
$$

Define $\widehat{M T}_{t}=\mathrm{N}^{2}\left(\frac{\mathrm{m}-1}{\mathrm{n}-1}\right)\left[\frac{\mathrm{N}-1}{\mathrm{~N}}\left(\frac{\mathrm{m}-2}{\mathrm{n}-2}\right)+\frac{1}{\mathrm{~N}}\right]$

Unbiased estimate of variance of Sum. $\mathrm{v}\left(\mathrm{T}_{t}\right)=$

$$
\widehat{\mathrm{T}}_{t}^{2}-\left[\widehat{M T}_{t}\left(\mathrm{Z}-\mathrm{s}^{2}\right)+\hat{\mathrm{M}}_{t} \mathrm{~s}_{t}^{2}\right]
$$

\section{Retain the best estimate}

If $\mathrm{t}=1$, store the estimates $\hat{\mathrm{M}}=\hat{\mathrm{M}}_{1}$, $\mathrm{v}(\hat{\mathrm{M}})=\mathrm{v}\left(\hat{\mathrm{M}}_{1}\right), \overline{\mathrm{x}}=\overline{\mathrm{x}}_{1}, \quad \widehat{\mathrm{T}}=\widehat{\mathrm{T}}_{1}, \mathrm{~s}^{2}=\mathrm{s}_{1}^{2}, \mathrm{v}(\overline{\mathrm{x}})=$ $\mathrm{v}\left(\overline{\mathrm{x}}_{1}\right), \widehat{M T}=\widehat{M T}_{1}$, and $\mathrm{v}(\widehat{\mathrm{T}})=\mathrm{v}\left(\widehat{\mathrm{T}}_{1}\right)$ as the best estimates of M, Sum and Average and their variances.

If $1<t \leq 10$ and the latest estimates $\mathrm{v}\left(\widehat{\mathrm{M}}_{t}\right)$, $\mathrm{v}\left(\overline{\mathrm{x}}_{t}\right)$ and $\mathrm{v}\left(\hat{\mathrm{T}}_{t}\right)$ of variances are smaller than the best estimates (among previous runs $1, \ldots, \mathrm{t}$ 1) then update the best estimates to the latest estimates with reference to current $t$ value. The user chooses to use one or more of variances $v(\hat{M}), \quad v(\bar{X})$ and $\quad v(\hat{T})$ to control the updating step depending upon the priority he has in estimating M, Average or Sum.

Set $\mathrm{t}=\mathrm{t}+1$

End Loop $\quad / /$ when $\mathrm{t}>10$

End

\section{EXPERIMENTAL RESULTS}

To evaluate the effectiveness of the proposed 
sampling technique based on the $k$-MDI tree, experiments were performed on real life supermarket retail sales data (TUN 2007) for a month from 150 outlets. The data warehouse is structured as a star schema shown in Figure 4, with the fact table (itemscan) consisting of over 21 million rows and three dimension tables viz. storeInfo, itemDesc and storeMemberVisits.

In Section 3, the concept of inverse random sampling without replacement (Chaudhuri and Mukerjee, 1985); (Sangngam and Suwatee, 2010) along with unbiased estimation techniques was introduced for estimating the mean, Sum and M, the count of records satisfying a property $\mathrm{P}$ in the database. Next, the results of the experiments are shown using the $k$-MDI tree to facilitate random retrieval of records to estimate $\mathrm{M}$.

We considered a set of three queries (modified version of TPC-H Query 1 (TPC-H 2007) containing the SQL functions $-\operatorname{avg}(), \operatorname{sum}(), \operatorname{count}()$ with varying database relevancy ratios (or DRR, as defined in Section 2.4), viz. low $(<0.01)$, medium $(0.01-0.1)$ and high $(>0.1)$ DRRs. The queries were of the form:

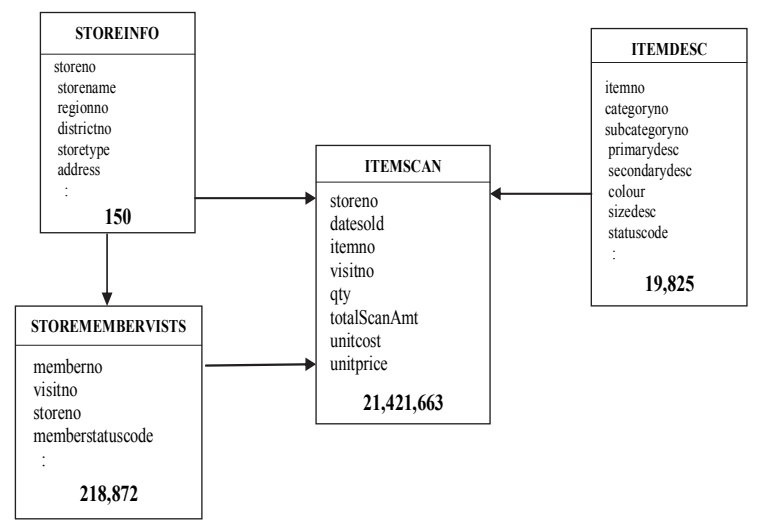

Figure 4: The schema for experimental retail sales data warehouse.

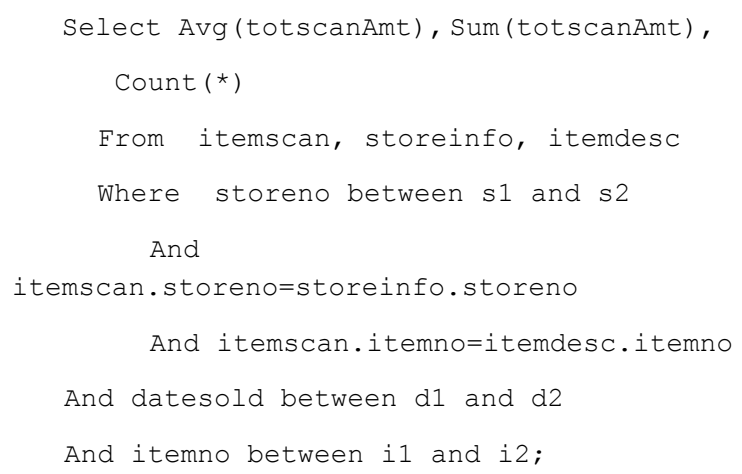

Table 2 shows the percentage error rates for low database relevancy ratio, using the 10 different sampling rates pertaining to 5 percentile values of $\rho(Q)$ at the 0.10 rate and 5 for 0.20 rate as per the sampling and estimation schemes discussed in Section 3. Here, $\mathrm{m}_{1,1}$ refers to 0.10 rate for the $10^{\text {th }}$ percentile value of $\rho(Q) ; \mathrm{m}_{1,2}$ for the $25^{\text {th }}$ percentile value; $\mathrm{m}_{1,3}$ for the $50^{\text {th }} ; \mathrm{ml}, 4$ for $75^{\text {th }}$; and $\mathrm{m}_{1,5}$ for $90^{\text {th }}$; while $m_{2,1}-m_{2,5}$ for the same percentile values respectively but at 0.20 rate. Table 2 and the corresponding graph in Figure 5 show the accuracy levels achieved by the sampling scheme using inverse SRSWOR for low DRR queries. It is observed that the error rates for calculation of Avg are below $5 \%$, but estimates of both the $\mathrm{M}$ and the Sum are not within the acceptable error limits of $5 \%$.

Table 2: Estimating for M, Avg and Sum using inverse SRSWOR - Low DRR

\begin{tabular}{|c|c|c|c|c|}
\hline $\begin{array}{c}\text { Sampling } \\
\text { Rate }\end{array}$ & $\begin{array}{c}\text { Est. value of } \\
\mathbf{M}\end{array}$ & $\begin{array}{c}\text { Error in } \\
\text { estimating M }\end{array}$ & $\begin{array}{c}\text { Error } \\
\text { Avg }\end{array}$ & $\begin{array}{c}\text { Error } \\
\text { Sum }\end{array}$ \\
\hline $\mathrm{m} 1,1$ & 3231 & 6.61 & 4.1 & 14.68 \\
\hline $\mathrm{m} 1,2$ & 2758 & 9.01 & 2.21 & 12.02 \\
\hline $\mathrm{m} 1,3$ & 2757 & 9.05 & 3.12 & 19.08 \\
\hline $\mathrm{m} 1,4$ & 3286 & 8.42 & 2.59 & 15.46 \\
\hline $\mathrm{m} 1,5$ & 3353 & 10.61 & 2.19 & 15.4 \\
\hline $\mathrm{m} 2,1$ & 2670 & 11.9 & 1.78 & 12.87 \\
\hline $\mathrm{m} 2,2$ & 3340 & 10.18 & 1.36 & 14.65 \\
\hline $\mathrm{m} 2,3$ & 3262 & 7.62 & 1.27 & 9.86 \\
\hline $\mathrm{m} 2,4$ & 2782 & 8.21 & 1.69 & 9.28 \\
\hline $\mathrm{m} 2,5$ & 3241 & 6.92 & 1.59 & 8.94 \\
\hline
\end{tabular}

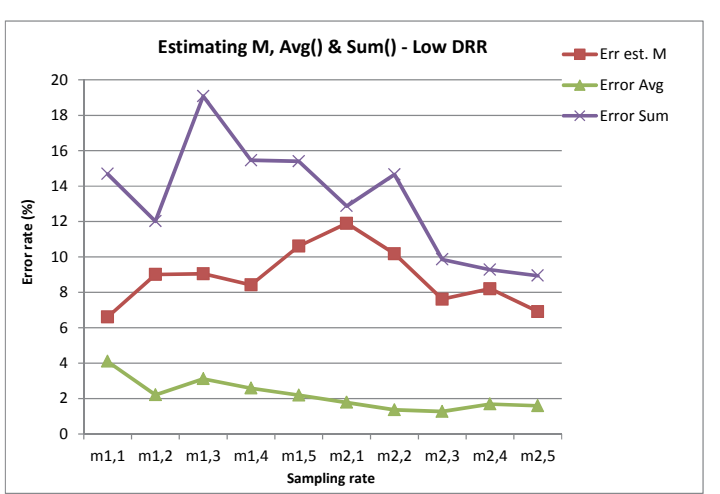

Figure 5: Error rates of estimating M, Avg and Sum using SRSWOR for low DRR.

Table 3 and the corresponding graph in Figure 6 show the accuracy levels achieved by the sampling scheme as described for inverse SRSWOR on medium DRR queries. It can be observed that for medium DRR queries, the count $M$, the Avg and the Sum are within the acceptable error rate limits of 0 $5 \%$. 
Table 3: Estimating for M, Avg and Sum using inverse SRSWOR - Medium DRR.

\begin{tabular}{|c|c|r|r|r|}
\hline $\begin{array}{c}\text { Sampling } \\
\text { Rate }\end{array}$ & Est. of $\mathbf{M}$ & Err est. M & Error Avg & Error Sum \\
\hline $\mathrm{m} 1,1$ & 11683 & 3.12 & 2.06 & 4.02 \\
\hline $\mathrm{m} 1,2$ & 11582 & 2.22 & 2.63 & 3.88 \\
\hline $\mathrm{m} 1,3$ & 11600 & 2.38 & 2.66 & 3.86 \\
\hline $\mathrm{m} 1,4$ & 11621 & 2.57 & 2.11 & 3.42 \\
\hline $\mathrm{m} 1,5$ & 11575 & 2.16 & 2.13 & 3.24 \\
\hline $\mathrm{m} 2,1$ & 11602 & 2.4 & 2.31 & 2.64 \\
\hline $\mathrm{m} 2,2$ & 11500 & 1.5 & 2.22 & 2.22 \\
\hline $\mathrm{m} 2,3$ & 11391 & 0.54 & 2.03 & 2.86 \\
\hline $\mathrm{m} 2,4$ & 11558 & 2.01 & 2.01 & 2.1 \\
\hline $\mathrm{m} 2,5$ & 11526 & 1.73 & 1.64 & 2.08 \\
\hline
\end{tabular}

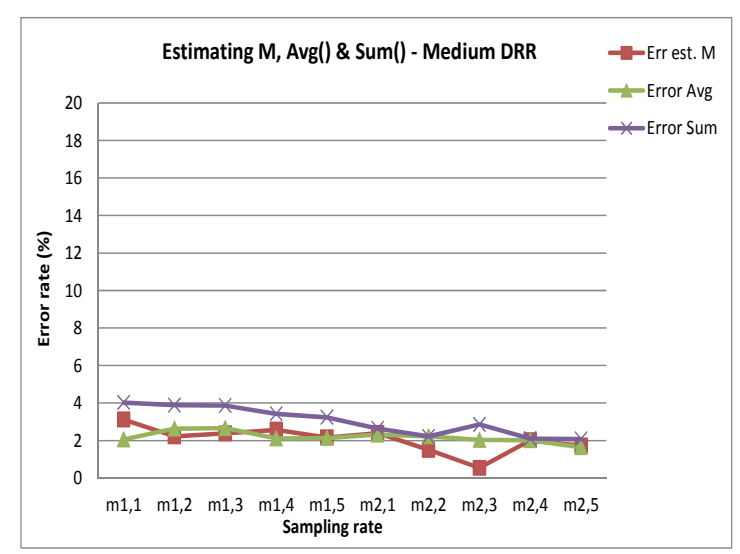

Figure 6: Error rates of estimating M, Avg and Sum using SRSWOR for medium DRR.

Table 4 and the corresponding graph in Figure 7 show the accuracy levels achieved by the sampling scheme for inverse SRSWOR on high DRR queries. It can be observed that the count M, the Avg and the Sum were quite accurately estimated as they are within the acceptable error rate limits of $0-5 \%$.

Table 4: Estimating for $\mathrm{M}$, Avg and Sum using inverse SRSWOR - High DRR

\begin{tabular}{|c|r|r|r|r|}
\hline Sampling Rate & Est. of $\mathbf{M}$ & Err est. $\mathbf{M}$ & Error Avg & Error Sum \\
\hline $\mathrm{m} 1,1$ & 329333 & 1.97 & 2.45 & 3.06 \\
\hline $\mathrm{m} 1,2$ & 331819 & 1.23 & 2.01 & 2.87 \\
\hline $\mathrm{m} 1,3$ & 342099 & 1.83 & 2.44 & 2.05 \\
\hline $\mathrm{m} 1,4$ & 339680 & 1.11 & 1.98 & 2.56 \\
\hline $\mathrm{m} 1,5$ & 328997 & 2.07 & 1.82 & 2.32 \\
\hline $\mathrm{m} 2,1$ & 329803 & 1.83 & 1.1 & 2.18 \\
\hline $\mathrm{m} 2,2$ & 329299 & 1.98 & 1.44 & 2.28 \\
\hline $\mathrm{m} 2,3$ & 331449 & 1.34 & 1.29 & 1.05 \\
\hline $\mathrm{m} 2,4$ & 339949 & 1.19 & 0.52 & 0.76 \\
\hline $\mathrm{m} 2,5$ & 339546 & 1.07 & 0.63 & 1.11 \\
\hline & & & & \\
\hline
\end{tabular}

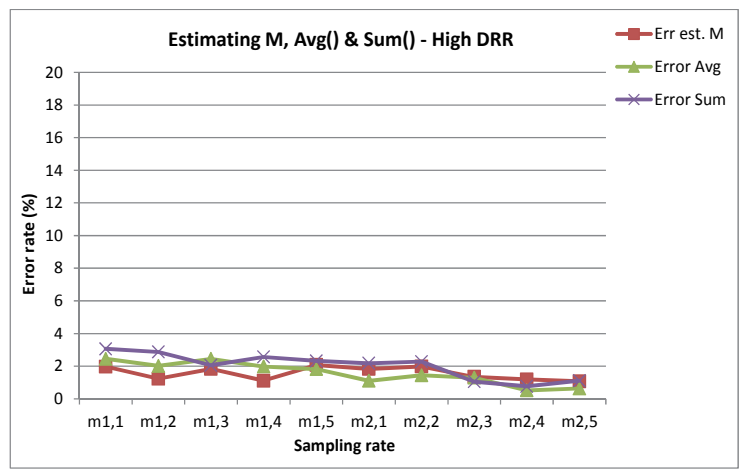

Figure 7: Error rates of estimating M, Avg and Sum using SRSWOR for high DRR.

In general, the accuracies achieved for high DRR queries are better than those of medium DRR. Thus, the sensitivities of the error rates for all the three statistics viz., AVG, SUM and $M$ are much less, i.e. show fewer fluctuations, as compared to that of the medium DRR.

\section{CONCLUSIONS}

In this paper, an innovative estimation scheme based on inverse simple random sampling without replacement (SRSWOR) was presented for approximate processing of data warehouse queries. Using this technique, the total number $M$ of records in the whole database satisfying the query conditions was estimated along with the mean and sum for typical queries. The $k$-MDI tree index was used to draw the samples efficiently. It was found that for queries of low database relevancy ratio (DRR), the estimated values of average were within the acceptable error limit of 5\%, but not the estimates of sum and the total number of relevant records $M$. However, for both medium and high DRR queries, all the three statistics viz. the value of $M$, the average and the sum were estimated with error rates below 5\% as shown in Section 4. Future research may incorporate the probabilistic approaches such as those in (Aouiche and Lemire 2007); (Heule et al., 2013 ) in our algorithm based on $k$-MDI tree.

\section{REFERENCES}

Aouiche, K. and Lemire, D. 2007. A Comparison of Five Probabilistic View-Size Estimation Techniques in OLAP, DOLAP'07, November 2007, Lisboa, Portugal. Bentley, J. L. 1975. Multidimensional binary search trees used for associative searching, Communications of the 
$A C M$, September 1975, 18(9): 509-517.

Berenson, M. L., and D. M. Levine. 1992. Basic Business Statistics - Concepts and Applications. Prentice Hall, Upper Saddle River, New Jersey, USA.

Chaudhuri, A., and R. Mukerjee. 1985. Domain Estimation in Finite Populations. Australian Journal of Statistics. 27(2): 135-137.

Chaudhuri, S. 2012. What Next? A Half-Dozen Data Management Research Goals for BigData and the Cloud. PODS 2012. May 21-23. Scottsdale, Arizona, USA.

Fisher, D. 2011. Incremental, Approximate Database Queries and Uncertainty for Exploratory Visualization. IEEE Symposium on Large Data Analsis and Visualization. 73-80. October 23-24. Providence, RI, USA.

Fisher, D., I. Popov, S. M. Drucker, and M. Schraefel. 2012. Trust Me, I'm Partially Right: Incremental Visualization Lets Analysts Explore Large Datasets Faster. CHI 2012, May 5-10. Austin, Texas, USA. 1673-1682.

Heule, S., Numkesser, M. and Hall, A. 2013. HyperLogLog in Practice: Algorithmic Engineering of a State of The Art Cardinality Estimation Algorithm. EDBT/ICDT'13 2013, March 18-22. Genoa, Italy.

Hobbs, L., S. Hillson, and S. Lawande. 2003. Oracle9iR2 Data Warehousing. Elsevier Science, MA, USA.

Jermaine, C., 2007. Random Shuffling of Large Database Tables. IEEE Transactions on Knowledge and Data Engineering. 18(1):73-84.

Jermaine, C. 2003. Robust Estimation with Sampling and Approximate Pre-Aggregation. VLDB Conference Proceedings 2003, 886-897.

Jermaine, C., A. Pol, and S. Arumugam. 2004. Online Maintenance of Very Large Random Samples. SIGMOD Conference Proceedings 2004.

Jin, R., L. Glimcher, C. Jermaine, and G. Agrawal. 2006. New Sampling-Based Estimators for OLAP Queries. Proceedings of the 22nd International Conference on Data Engineering (ICDE'06), Atlanta, GA, USA.

Joshi, S., and C. Jermaine. 2008. Matirialized Sample Views for Database Approximation, IEEE Transcaions on Knowledge and Data Engineering, 20:3 pp. 337-351.

Li, X., J. Han, Z. Yin, J-G. Lee, and Y. Sun. 2008. Sampling Cube: A Framework for Statistical OLAP over Sampling Data. Proceedings of ACM SIGMOD International Conference on Management of Data (SIGMOD'08), Vancouver, BC, Canada, June.

Olken, F., and D. Rotem. 1990. Random Sampling from Database File. In: A Survey. International Conference on Scientific and Statistical Database Management, 1990. pp. 92-111.

Rudra, A., R. Gopalan and N.R. Achuthan. 2012. Efficient Sampling Techniques in Approximate Decision Support Query Processing. Proceedings of the International Conference on Enterprise Information Systems - ICEIS 2012, Wroclaw, Poland. June 28-July 22012.

Sangngam, P., and P. Suwatee. 2010. Modified Sampling
Scheme in Inverse Sampling without Replacement. 2010 International Conference on Networking and Information Technology. IEEE Press, New York, USA. 580-584.

Spiegel, J., and N. Polyzotis. 2009. TuG Synopses for Approximate Query Answering. ACM Transactions on Database Systems. (TODS) 34(1).

TUN. 2007. Teradata University Network. http://www.teradata.com/TUN databases. (accessed June 12, 2007).

TPC-H. 2007. Transaction Processing Council. Decision Support Queries. http://www.teradata.com/ TUN_databases. (accessed April 23, 2007). 